\title{
The Age Dependent Progression of Hajdu-Cheney Syndrome in Two Families
}

Jitka Jirečková1, Martin Magner', Lukáš Lambert'², Alice Baxová3, Alena Leiská ${ }^{2}$, Lenka Kopečková ${ }^{4}$, Lenka Fajkusová ${ }^{4}$, Jiří Zeman ${ }^{1}$ ${ }^{1}$ Department of Pediatrics and Adolescent Medicine, First Faculty of Medicine, Charles University and General University Hospital in Prague, Prague, Czech Republic;

${ }^{2}$ Department of Radiology, First Faculty of Medicine, Charles University and General University Hospital in Prague, Prague, Czech Republic; ${ }^{3}$ Institute of Biology and Medical Genetics, First Faculty of Medicine, Charles University and General University Hospital in Prague, Prague, Czech Republic; ${ }^{4}$ Centre of Molecular Biology and Gene Therapy, Masaryk University in Brno and University Hospital Brno, Brno, Czech Republic

Received November 28, 2018; Accepted January 31, 2019.

Key words: Hajdu-Cheney syndrome - Short stature - Wormian bones Acro-osteolysis

Abstract: Hajdu-Cheney syndrome (HCS) is a rare multi-system disease with autosomal dominant inheritance and skeletal involvement, resulting mostly in craniofacial dysmorphy with mid-face hypoplasia, dental anomalies, short stature, scoliosis, shortening of the digits and nail beds, acro-osteolysis and osteoporosis. We report the progression of clinical and radiographic findings in five patients with Hajdu-Cheney syndrome from two families. A custom capture array designed to capture exons and adjacent intron sequences of 230 selected genes were used for molecular analyses, and the pathogenic variants identified were confirmed by PCR and Sanger sequencing. In both families we observed age-dependent changes in the disease, with a progression of pain in older patients, a shortening of digits and nail

This study was supported by projects of the Ministry of Health (RVO-VFN 64165/2012) and the Ministry of Education, Youth, and Sports of the Czech Republic (PROGRES Q32/LF2).

Mailing Address: Prof. jiř́ Zeman, MD., PhD., Department of Pediatrics and Adolescent Medicine, First Faculty of Medicine, Charles University and General University Hospital in Prague, Ke Karlovu 2, 12808 Prague 2, Czech Republic; Phone: +420 224967 791; e-mail: jzem@lf1.cuni.cz 
beds on both the hands and feet, kyphoscoliosis and the persistence of Wormian bones in lambdoid sutures. Molecular analyses performed in two patients revealed that they are heterozygotes for a c.6255T>A (p.Cys2085*) variant in the NOTCH2 gene, resulting in a premature stop-codon. Bone mineral density $(Z$-score $<-2)$ did not improved in a girl treated with calcium and vitamin $D$ supplementation during childhood and bisphosphonate during adolescence. Hajdu-Cheney syndrome is a slowly progressive disease with a frequently unfavourable prognosis in elderly patients, especially for the development of dental anomalies, osteoporosis and the progression of skeletal complications requiring orthopedic surgeries.

\section{Introduction}

Hajdu-Cheney syndrome (HCS) is a rare multi-system disease with skeletal involvement and autosomal dominant inheritance, and is associated with mutations in the terminal exon 34 of the NOTCH2 gene encoding the transmembrane $\mathrm{NOTCH} 2$ receptor (Isidor et al., 2011; Simpson et al., 2011; Adami et al., 2016; Pittaway et al., 2018). The disease is slowly progressive with an onset in childhood or adolescence resulting in short stature, craniofacial dysmorphism with mid-face hypoplasia, hypertelorism, bushy eyebrows, micrognathia, small mouth and dental anomalies, vertebral anomalies, scoliosis, and bowing of the long bones. Multiple Wormian bones in scull sutures and acro-osteolysis of the distal phalanges on X-ray and osteoporosis are typical findings. Other features may develop, including hearing loss, renal cysts and cardiovascular involvement (Ramos et al., 1998; Isidor et al., 2011; Regev et al., 2019).

Here we present the age-dependent progression of clinical and radiological manifestations of Hajdu-Cheney syndrome in five patients from two families.

\section{Methods}

DNA was isolated from peripheral blood. For molecular genetic diagnostics of skin, bone, and connective tissue disorders, we developed a solution-based capture method using the SeqCap EZ Choice Library (Roche NimbleGen, Madison, WI, USA) and targeted sequencing on a NextSeq (Illumina, San Diego, CA, USA). Sequencing data were evaluated using the software Sequence Pilot (JSI Medical Systems, Kippenheim, Germany). A custom capture array was designed to capture exons and adjacent intron sequences of 230 selected genes, among them the gene $\mathrm{NOTCH} 2$ associated with Hajdu-Cheney syndrome. Identified pathogenic variants were confirmed by PCR and Sanger sequencing using the BigDye Terminator Cycle Sequencing Kit (Applied Biosystems Foster City, CA, USA) on an ABI 3130xl Genetic Analyzer (Applied Biosystems).

\section{Ethics}

The study was approved by the Ethics Committee of the General University Hospital in Prague and was conducted in agreement with the Declaration of Helsinki. Written 
informed consent for molecular analyses was obtained from a 19-year-old girl and a 17-year-old boy and his mother.

\section{Patients}

The proband (P1) from the first family is a 17-year-old boy with a behavioural disturbance, ADHD (attention deficit hyperactivity disorder) and border low mental capacity (IQ 76-80), short stature $\left(167 \mathrm{~cm}, 3^{\text {rd }}\right.$ percentile), mid-face hypoplasia with bushy eyebrows, synophrys, down-slanted palpebral fissures, micrognathia, a mixed set of teeth with the persistence of one milk tooth and the delayed eruption of two teeth, hypermobility of large joints, kyphoscoliosis, bowed legs, dyskeratosis, and a mild shortening of several nail beds since infancy (Figure 1). During early childhood he had frequent upper respiratory infections and recurrent warts on his palms and soles regardless of therapy that including surgery and liquid nitrogen. His growth percentile gradually declined from the $40^{\text {th }}$ percentile at birth to the $10^{\text {th }}$ percentile at the age of 10 years and the $3^{\text {rd }}$ percentile after puberty. The bone mineral density
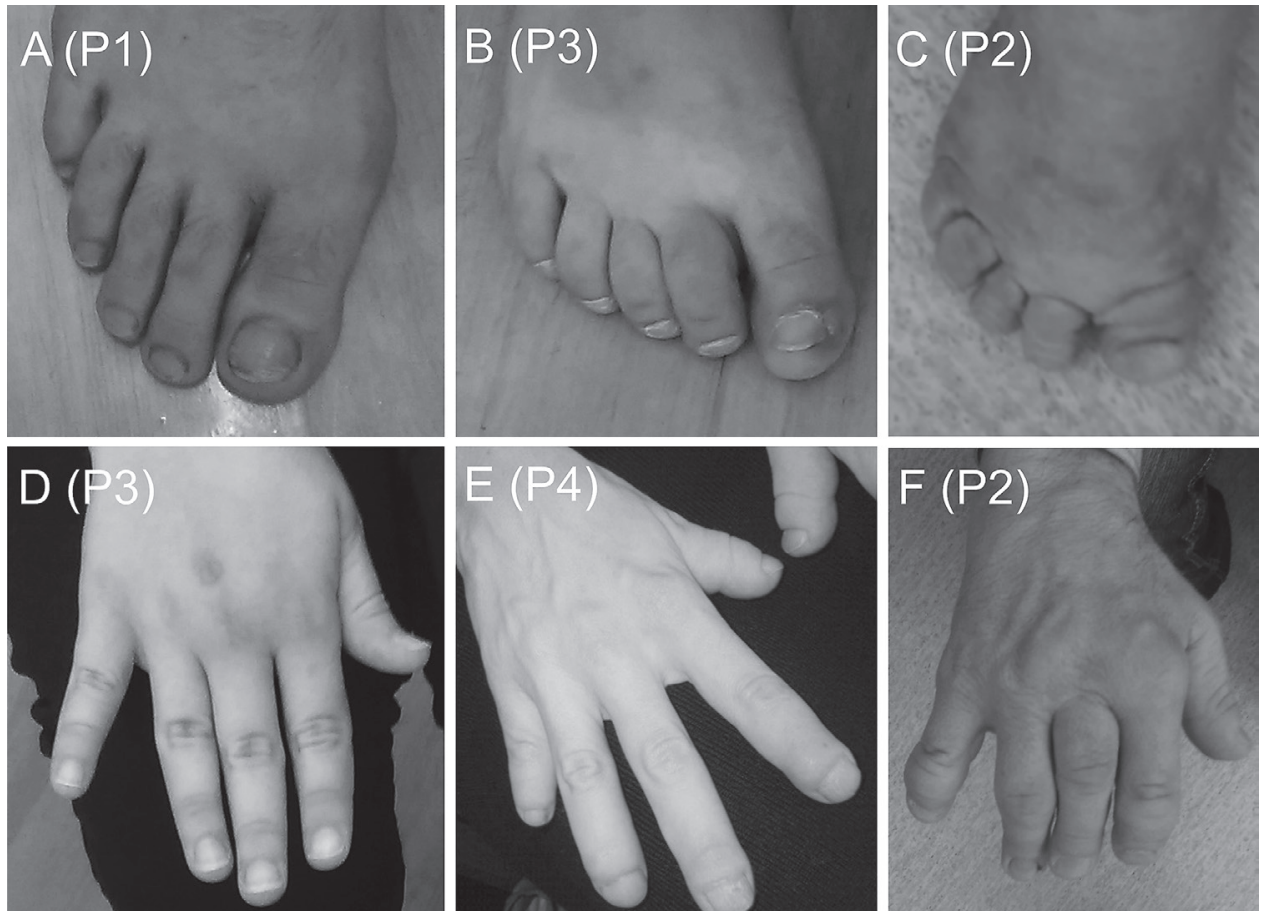

Figure 1 - Photographs of feet (above) and hands (below) in patients with Hajdu-Cheney syndrome: (A) mild shortening of the nail beds on the foot of a 17-year-old boy (P1); (B) shortening of several nails in his 19-year-old sister (P3); (C) very short digits and nails in their 55-year-old father (P2); (D) nearly normal digits on the hand of the 19-year-old girl (P3); (E) very short digits and nails in her father (P2); (F) shortening of digits $I$ and $V$ in a 24-year-old women (P4). 

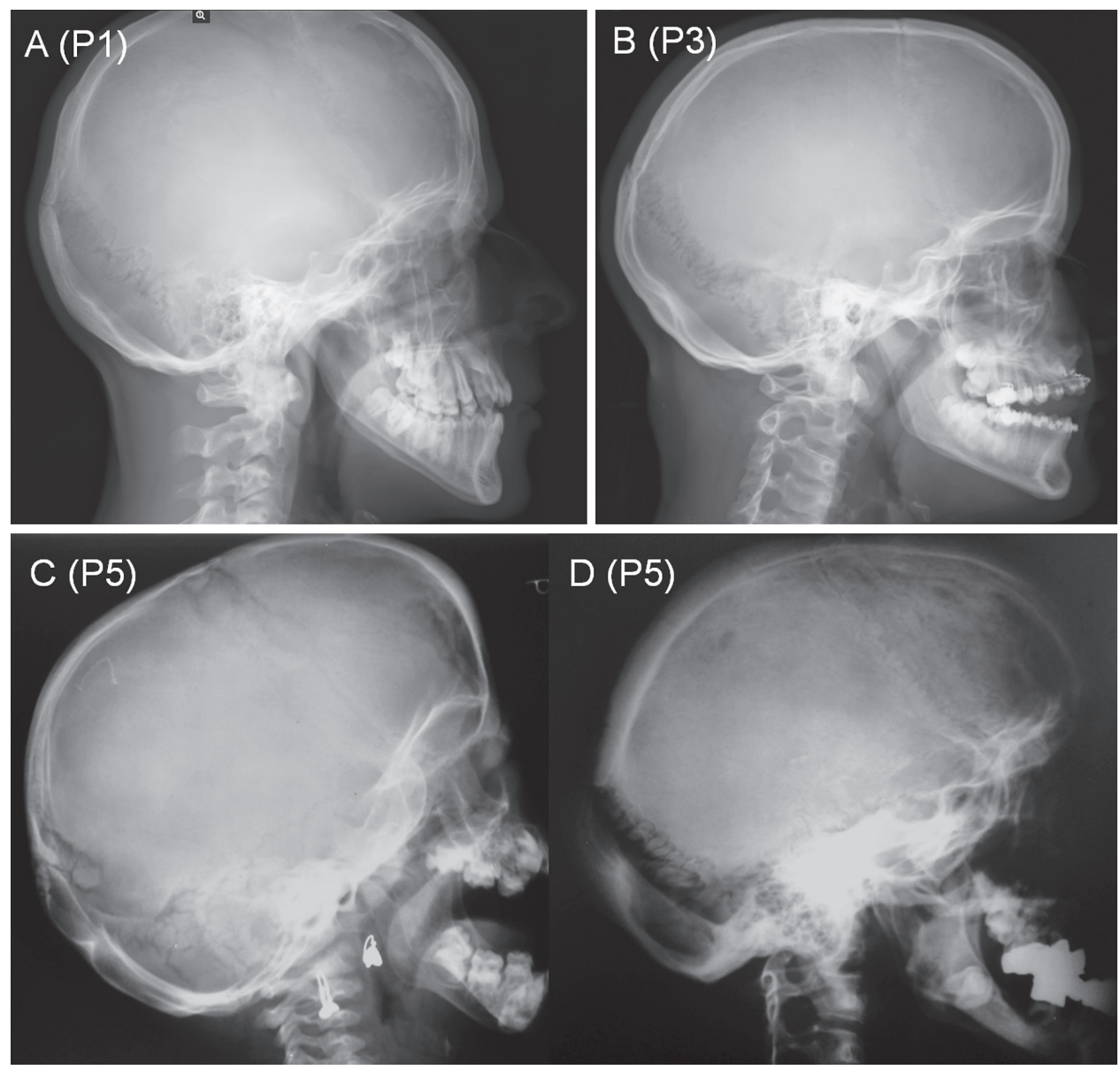

Figure 2 - Wormian bones in the lambdoid sutures, dental abnormalities and hypoplasia of frontal cavities in patients with Hajdu-Cheney syndrome: (A) a 17-year-old boy (P1); (B) a 19-year-old girl (P3) and progression of the disease in the girl (P5) from the age of 3.5 years (C) to the age of 15 years (D).

(BMD) of the lumbar spine is decreased (Z-score -1.6). An X-ray survey revealed multiple Wormian bones in the lambdoid suture, dental abnormalities, hypoplasia of the frontal sinuses (Figure 2) and acro-osteolysis on toes I-III and all fingers (Figure 3).

His father (P2) is a 55-year-old man with short stature $\left(165 \mathrm{~cm},<3^{\text {rd }}\right.$ percentile) and sore fingers on both hands and feet since the second decade of his life, progressively resulting in acquired deformities and a profound shortening of most of the fingers and the loss of several nails (Figure 1). He has repeatedly been seen by several specialists and treated with antibiotics and anti-inflammatory drugs without any success. He has increasing problems with sore joints on the hands and feet, has 
160) Prague Medical Report / Vol. 119 (2018) No. 4, p. 156-164

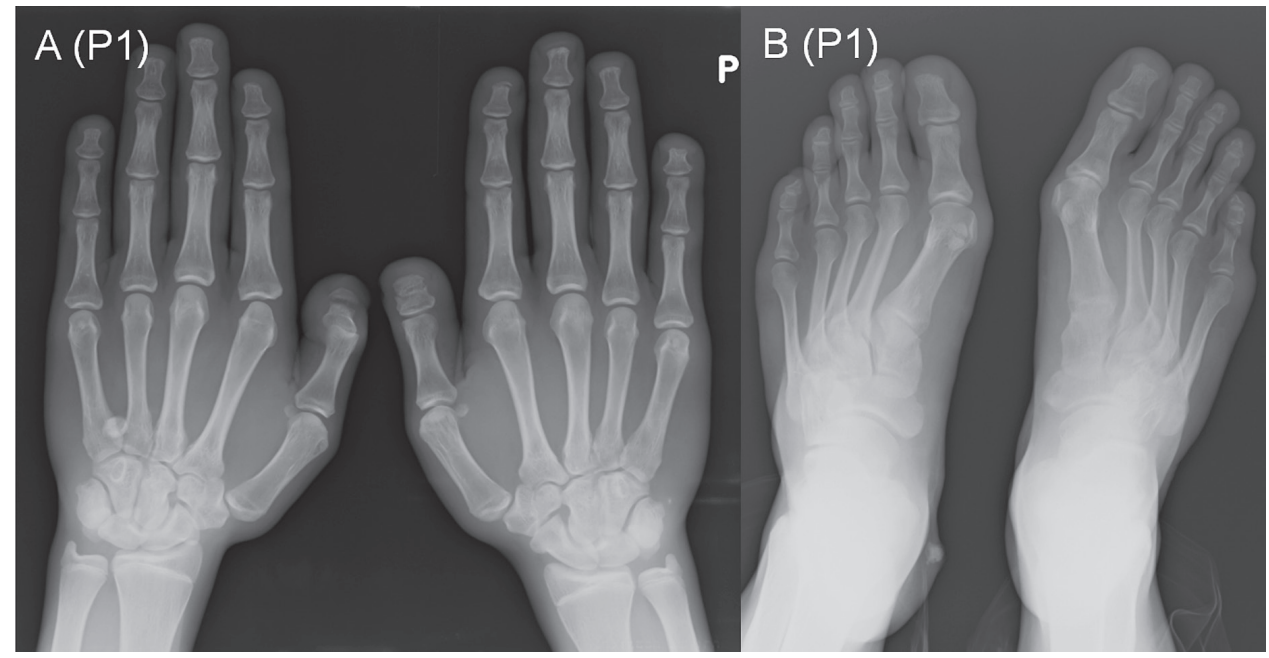

Figure 3 - Acro-osteolysis of all distal phalanges on the hands (A) and distal phalanges I-III on the feet (B) of a 17-year-old boy with Hajdu-Cheney syndrome (P1).

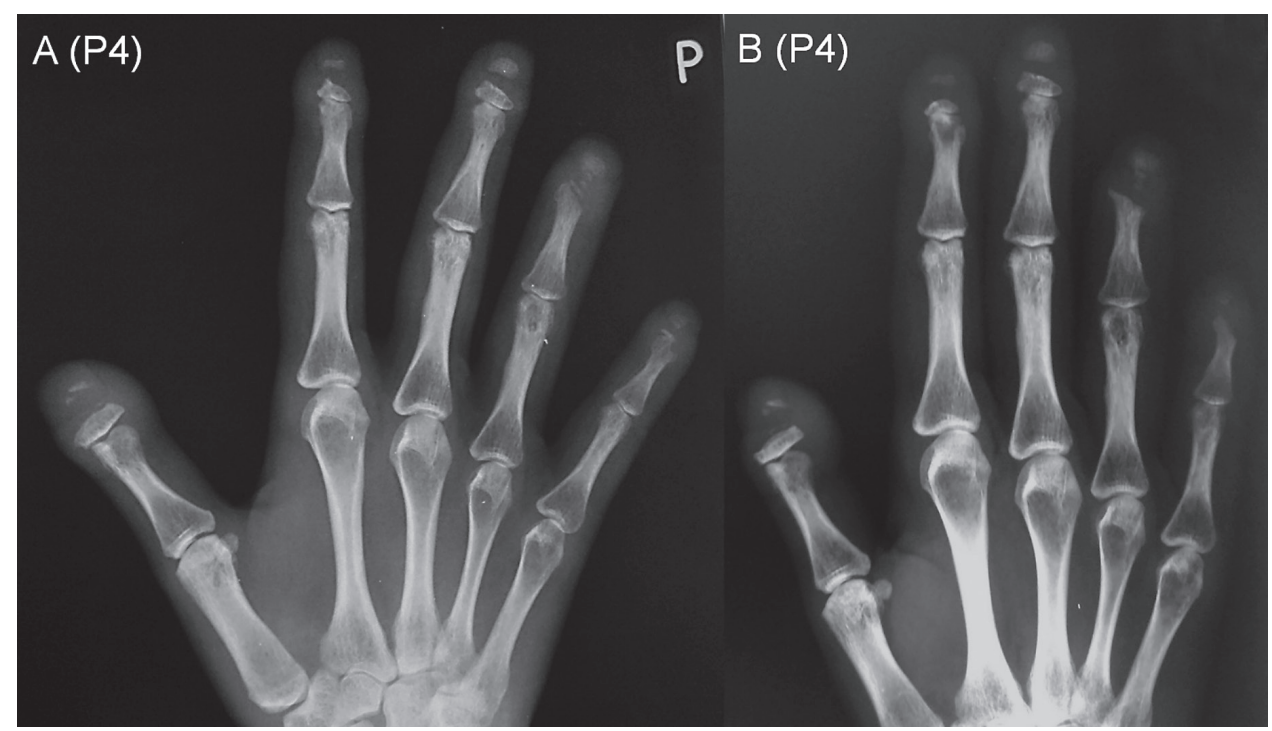

Figure 4 - Progression of acro-osteolysis in patient with Hajdu-Cheney syndrome (P4) from the age of 24 years (A) to the age of 40 years (B).

flat feet, and he is unable to walk longer distances. The soft tissue and skin on several fingers are frilled. He has normal dentition and wears glasses for hyperopia.

The proband's sister (P3) is a 19-year-old girl with low stature $\left(155 \mathrm{~cm}, 3^{\text {rd }}\right.$ percentile). She has severe myopia (contact lenses -7 D), mild mid-face hypoplasia 


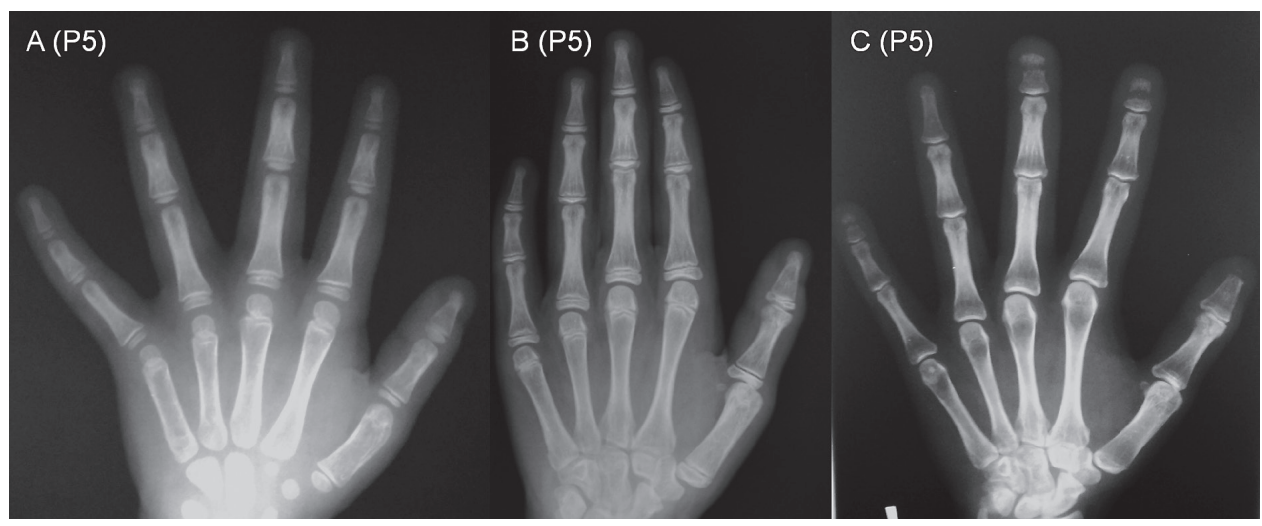

Figure 5 - Progression of acro-osteolysis in patient with Hajdu-Cheney syndrome (P5) at the age of 8 (A), 14 (B) and $16(C)$ years.

and fixed braces after surgery of the upper jaw. Further abnormities include genua valga, flat feet, and several short nails on her feet (Figure 1). An X-ray revealed multiple Wormian bones in the lambdoid suture, dental abnormalities and hypoplasia of the frontal sinuses (Figure 2).

The second family was partly reported in 1994 (Zeman et al., 1994). At the age of 24 years the proband (P4) had progressive back pain, short stature, mid-face hypoplasia, dental anomalies, shortening of several digits (Figure 1) with acroosteolysis on both hands and Wormian bones in the lambdoid suture. She required several spine surgeries because of severe kyphoscoliosis, used a wheel-chair since the end of her fourth decade, and died at the age of 43 years.

Her daughter (P5) had hypertelorism and normal X-rays of her hands at the age of 2.5 years, but her first acro-osteolysis appeared a year later. In addition, Wormian bones in the lambdoid sutures were observed at the age of 3.5 years. Since the age of 13 years, she has had acro-osteolysis on most fingers of both hands (Figure 5). She has also developed kyphoscoliosis and underwent neurosurgery because of progressive headaches due to a Chiari II malformation with basilar impression at the age of 14 years, and surgery for dentition abnormalities one year later. Currently she has short stature $\left(148 \mathrm{~cm},<3^{\text {rd }}\right.$ percentile), and the BMD of her lumbar spine and femoral neck has repeatedly been in the range indicating osteoporosis (Z-score $<-2$ ) despite therapy with bisphosphonates, calcium and vitamin D supplementation.

\section{Results}

The results of molecular analyses demonstrated that patients $\mathrm{P} 1$ and $\mathrm{P} 2$ are heterozygotes for the novel c.6255T>A (p.Cys2085*) variant in the NOTCH2 gene resulting in a premature stop-codon. The mutation is not described in the databases HGMD $^{\circledR}$ Professional 2018.4 and ClinVar. DNA from the members of the second family (P4, P5) is not available. 
The disease progression with the age-dependent shortening of digits and nails on the feet in patients P1-3 and the hands in patients P2-4 are shown on Figure 1. Radiographic findings in patients P1 and P3 revealed skull abnormalities with Wormian bones in the lambdoid sutures, dental abnormalities and hypoplasia of the frontal sinuses (Figure 2A and B). The progression of skull abnormalities in P5 during childhood is shown in Figure 2C and D. Acro-osteolysis of all distal phalanges on the hands and three distal phalanges on the feet of $\mathrm{P} 1$ at the age of 17 years are shown on Figure 3. Figures 4 and 5 document the development of acro-osteolysis in P4 between the age of 24 and 40 years, and P5 during her childhood and adolescence, respectively.

\section{Discussion}

A new type of cranio-skeletal dysplasia was described in 1948 by Hajdu and Kauntze, and since 1965 has been reported as Hajdu-Cheney syndrome (Hajdu and Kauntze, 1948; Cheney, 1965). The disease is caused by the mutation in exon 34 of the NOTCH2 gene, encoding the transmembrane $\mathrm{NOTCH} 2$ receptor involved in the coupled processes of bone formation and resorption (Pittaway et al., 2018). This NOTCH2 mutation leads to the premature truncation of the transmembrane NOTCH2 receptor, with either the disruption or loss of the C-terminal prolineglutamate-serine-threonine-rich proteolytic recognition sequence with enhanced NOTCH2 signaling activity (Simpson et al., 2011; Canalis and Zanotti, 2014). In a mouse model of Hajdu-Cheney syndrome with the mutation $6955 \mathrm{C}>\mathrm{T}$ in NOTCH2 resulting in a Q2319X change at the amino acid level, bone osteopenia, enhanced osteoclastogenesis and increased bone resorption were found (Canalis et al., 2016).

Diagnosis of Hajdu-Cheney syndrome is based on clinical suspicion in combination with the results of $\mathrm{X}$-ray survey and molecular analyses. Although multiple Wormian bones and acro-osteolysis are quite typical for patients with Hajdu-Cheney syndrome, they are not sensitive enough for the diagnostics, because acro-osteolysis may clinically manifest only with minimal nails or skin changes and the radiographic findings of Wormian bones and acro-osteolysis may also occur in patients with other inherited or acquired diseases. For example, multiple Wormian bones are common in infants with osteogenesis imperfecta and acro-osteolysis may be found in patients with pycnodysostosis, severe psoriasis, systemic sclerosis, Raynaud's disease or other chronic vascular disturbances. The age of patients with Hajdu-Cheney syndrome at the onset of the first acro-osteolysis, which was documented by the X-ray, is variable, from one year (Gong et al., 2017) to the second or third decade. Acroosteolysis in our youngest patient (P5) was documented at the age of 3.5 years, while patient P3 still has no acro-osteolysis in her adolescence. Renal abnormalities, especially the polycystic kidney disease may be found approximately in $10 \%$ of patients with Hajdu-Cheney syndrome, but end-stage renal disease is rare (Battelino et al., 2016). So far, the renal functions in our patients P1 and P2 are normal. 
The prognosis of patients with Hajdu-Cheney syndrome is unfavorable and therapy is symptomatic, especially after the development of dental and orthopedic complications. The response to bisphosphonate treatment in preventing and/or treating already existent osteoporosis is variable and age-related, but probably less effective with advancing age (Galli-Tsinopoulou et al., 2012; Adami et al., 2016; Pittaway et al., 2018). Therapy with denosumab may increase bone mineral density, but does not prevent the progression of acro-osteolysis (Adami et al., 2016). The Z-score of bone mineral density did not improve in our patient (P5) treated with calcium and vitamin $\mathrm{D}$ supplementation during childhood and bisphosphonate during adolescence.

\section{Conclusion}

Hajdu-Cheney syndrome is a slowly progressive disease with usually an unfavorable prognosis in the elderly, mainly due to the development of dental anomalies, osteoporosis, and the progression of severe skeletal complications requiring orthopedic surgeries.

\section{References}

Adami, G., Rossini, M., Gatti, D., Orsolini, G., Idolazzi, L., Viapiana, O., Scarpa, A., Canalis, E. (2016) Hajdu Cheney syndrome; report of a novel NOTCH2 mutation and treatment with denosumab. Bone 92, 150-156.

Battelino, N., Writzl, K., Bratanič, N., Irving, M. D., Novljan, G. (2016) End-stage renal disease in an infant with Hajdu-Cheney syndrome. Ther. Apher. Dial. 20(3), 318-321.

Canalis, E., Zanotti, S. (2014) Hajdu-Cheney syndrome: a review. Orphanet J. Rare Dis. 9, 200.

Canalis, E., Schilling, L., Yee, S. P., Lee, S. K., Zanotti, S. (2016) Hajdu Cheney mouse mutants exhibit osteopenia, increased osteoclastogenesis and bone resorption. J. Biol. Chem. 291, 1538-1551.

Cheney, W. D. (1965) Acro-osteolysis. Am. J. Roentgenol. Radium Ther. Nucl. Med. 94, 595-607.

Galli-Tsinopoulou, A., Kyrgios, I., Giza, S., Giannopoulou, E. Z., Maggana, I., Laliotis, N. (2012) Two-year cyclic infusion of pamidronate improves bone mass density and eliminates risk of fractures in a girl with osteoporosis due to Hajdu-Cheney syndrome. Minerva Endocrinol. 37, 283-289.

Gong, R. L., Wu, J., Chen, T. X. (2017) A novel mutation of Notch homolog protein 2 gene in a Chinese family with Hajdu-Cheney syndrome. Chin. Med. J. (Engl.) 130, 2883-2884.

Hajdu, N., Kauntze R. (1948) Cranio-skeletal dysplasia. Br. J. Radiol. 21, 42-48.

Isidor, B., Lindebaum, P., Pichon, O., Bézieau, S., Dina, C., Jacquemont, S., Martin-Coignard, D. (2011) Truncating mutations in the last exon of $\mathrm{NOTCH} 2$ cause a rare skeletal disorder with osteoporosis. Nat. Genet. 43, 306-308.

Pittaway, J. F. H., Harrison, C., Rhee, Y., Holder-Espinasse, M., Fryer, A. E., Cundy, T., Drake, W. M., Irving, M. D. (2018) Bisphosphonate therapy for spinal osteoporosis in Hajdu-Cheney syndrome - new data and literature review. Orphanet J. Rare Dis. 13, 47.

Ramos, F. J., Kaplan, B. S., Bellah, R. D., Zackai, E. H., Kaplan, P. (1998) Further evidence that the HajduCheney syndrome and the "serpentine fibula-polycystic kidney syndrome" are a single entity. Am. J. Med. Genet. 78, 474-481.

Regev, M., Pode-Shakked, B., Jacobson, J. M., Raas-Rotschield, A., Goldstein, D. B., Anikster, Y. (2019) Phenotype variability in Hajdu-Cheney syndrome. Eur. J. Med. Genet. 62(1), 35-38. 
164) Prague Medical Report / Vol. 119 (2018) No. 4, p. 156-164

Simpson, M. A., Irving, M. D., Asilmaz, E., Gray, M. J., Dafou, D., Elmslie, F. V., Mansour, S., Holder, S. E., Brain, C., Burton, B. K., Kim, K. H., Pauli, R. M., Aftimos, S., Stewart, H., Kim, C. A., Holder-Espinasse, M., Robertson, S. P., Drake, W. M., Trembath, R. C. (2011) Mutations in NOTCH2 cause Hajdu-Cheney syndrome, a disorder of severe and progressive bone loss. Nat. Genet. 43, 303-305.

Zeman, J., Houstkova, H., Kozlowski, K. (1994) Hajdu-Cheney syndrome in a $31 / 2$ year old girl. Australas. Radiol. 38(3), 228-230.

Jirečková J.; Magner M.; Lambert L.; Baxová A.; Leiská A.; Kopečková L.; Fajkusová L.; Zeman J. 